\title{
Topological charge renormalization: A test case for 3-loop vacuum calculations using overlap fermions and Symanzik improved gluons
}

\author{
Apostolos Skouroupathis* \\ Univ of Cyprus; Physics Dept \\ E-mail: php4as01@ucy.ac.cy \\ Haralambos Panagopoulos \\ Univ of Cyprus; Physics Dept \\ E-mail: haris@ucy.ac.cy
}

\begin{abstract}
We calculate perturbative renormalization properties of the topological charge, using the standard lattice discretization given by a product of twisted plaquettes. We use the overlap and clover action for fermions, and the Symanzik improved gluon action for 4- and 6-link loops.

We compute the multiplicative renormalization of the topological charge density to one loop; this involves only the gluon part of the action. The power divergent additive renormalization of the topological susceptibility is calculated to 3 loops.
\end{abstract}

XXIIIrd International Symposium on Lattice Field Theory

25-30 July 2005

Trinity College, Dublin, Ireland

\footnotetext{
* Speaker.
} 


\section{Introduction}

Topological properties of QCD are among those most widely studied on the lattice. Various methods have been used to this end, involving renormalization, cooling, fermionic zero modes, geometric definitions, etc. In recent years, the advent of fermionic actions, such as the overlap, which do not violate chirality, has brought a new thrust to the subject.

In this work we compute the renormalization constants which are necessary in order to extract topological properties, in the "field theoretic" approach, from Monte Carlo simulations using Wilson or Symanzik improved gluons, and clover or overlap fermions. We compute the multiplicative renormalization $\mathbf{Z}_{Q}$ of the topological charge density, to 1 loop in perturbation theory, and the power divergent additive renormalization $\mathbf{M}\left(g^{2}\right)$ of the topological susceptibility, to 3 loops.

The main motivations for doing this work are: a) To enable comparison between different approaches used in studying topology, so that a coherent picture of topology in QCD may emerge. b) To enable studies, in numerical simulations, of quantities involving the density of topological charge, $q(x)$, rather than only the integrated charge; this is necessary, e.g., for studying the spin content of nucleons. c) As a feasibility study in lattice perturbation theory: Indeed, this is the first 3-loop calculation to appear in the literature, involving overlap fermions.

A more extensive write-up of this work can be found in our Ref. [1].

\section{Computation of $\mathbf{Z}_{Q}$}

Our first task is to compute the multiplicative renormalization $Z_{Q}$ 沟] of the topological charge density $q_{L}(x)$ to one loop, using the background field method. We use the standard definition of $q_{L}$, given by a product of twisted plaquettes

$$
q_{L}(x)=-\frac{1}{2^{9} \pi^{2}} \sum_{\mu \nu \rho \sigma= \pm 1}^{ \pm 4} \varepsilon_{\mu v \rho \sigma} \operatorname{Tr}\left[\Pi_{\mu v}(x) \Pi_{\rho \sigma}(x)\right]
$$

$\left(\varepsilon_{-\mu, v, \rho, \sigma} \equiv-\varepsilon_{\mu, v, \rho, \sigma} ;\right.$ in standard notation: $\Pi_{\mu v}(x)=U_{\mu}(x) U_{v}(x+\mu) U_{\mu}^{\dagger}(x+v) U_{v}^{\dagger}(x)$. $)$

The classical limit of the operator shown in Eq.(2.1) must be corrected by including a renormalization function $Z_{Q}$, which can be expressed perturbatively as

$$
Z_{Q}=1+Z_{1} \cdot g^{2}+\cdots, \quad Z_{1}=Z_{11} \cdot N_{c}+Z_{12} / N_{c}
$$

We perform a calculation of $Z_{1}$; this involves only the gluon part of the action.

In the background field method, link variables are decomposed as

$$
U_{\mu}(x)=V_{\mu}(x) U_{c \mu}(x), \quad V_{\mu}(x)=e^{i g Q_{\mu}(x)}, \quad U_{c \mu}(x)=e^{i a B_{\mu}(x)}
$$

in terms of links for a quantum field and a classical background field, respectively.

The diagrams involved in the one-loop calculation of $Z_{Q}$ are shown in Figure 1.

The standard Symanzik improved gauge field action, with 4- and 6-link Wilson loops, is

$$
\begin{aligned}
S_{G}=\frac{2}{g^{2}} & {\left[c_{0} \sum_{\text {plaquette }} \operatorname{Re} \operatorname{Tr}\left(1-U_{\text {plaquette }}\right)+c_{1} \sum_{\text {rectangle }} \operatorname{Re} \operatorname{Tr}\left(1-U_{\text {rectangle }}\right)\right.} \\
& \left.+c_{2} \sum_{\text {chair }} \operatorname{Re} \operatorname{Tr}\left(1-U_{\text {chair }}\right)+c_{3} \sum_{\text {parallelogram }} \operatorname{Re} \operatorname{Tr}\left(1-U_{\text {parallelogram }}\right)\right]
\end{aligned}
$$




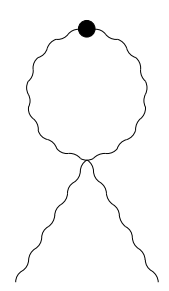

(a)

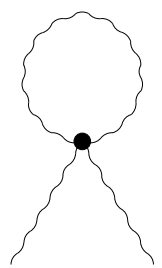

(b)

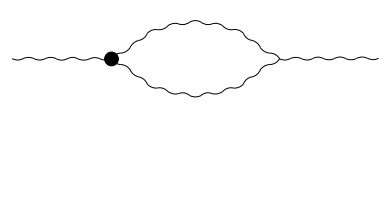

(c)

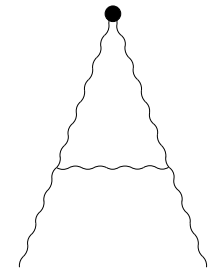

(d)

Figure 1: Diagrams contributing to $Z_{1}$. The bullet stands for topological charge vertices.

The Symanzik coefficients $c_{i}$ must satisfy: $c_{0}+8 c_{1}+16 c_{2}+8 c_{3}=1$.

Our calculations are performed without assumptions on the values of the external momenta $p_{1}, p_{2}$ : This is safest for $q_{L}$, otherwise one may easily end up with indeterminate expressions; it entails handling 3-point form factors [3] in $D=4-2 \varepsilon$ dimensions, such as:

$$
\bar{C}_{\mu v}\left(a, p_{1}, p_{2}\right)=\frac{(k a)^{2 \varepsilon}}{a^{0}} \int \frac{d^{D} k}{(2 \pi)^{D}} \frac{\sin _{\mu} \sin k_{v}}{\hat{k}^{2}\left(\widehat{k+a p_{1}}\right)^{2}\left(k+\widehat{a p_{1}+a p_{2}}\right)^{2}}
$$

Diagrams (c) and (d) of Figure 1, taken separately, exhibit poles in $\varepsilon\left(\right.$ (d) $\left.\propto-1 / \varepsilon-\ln \kappa^{2} a^{2}\right)$. These cancel, however, upon summation, as is expected by the fact that $Q$ does not renormalize in the continuum. The calculation of $Z_{Q}$ is particularly involved in the present case, involving propagators and vertices from the improved gluonic action. In particular, the calculation of diagram (d) involves a summation of $>1000000$ different algebraic expressions at intermediate stages.

Our results for $Z_{Q}$ are listed in Table 1 . In all calculations that involve the parameters $c_{i}$, we choose a standard set of values, as in Ref. [4]. The choice of the sets of parameters correspond to the most popular actions: The first set corresponds to the plaquette action, the second set corresponds to the tree-level Symanzik improved action [5] and the next 6 sets correspond to the tadpole improved Lüscher-Weisz (TILW) action [6] for 6 values of beta: $\beta=8.60,8.45,8.30,8.20,8.10,8.00$. The last two sets correspond to the Iwasaki [7] and DBW2 [8] actions, respectively.

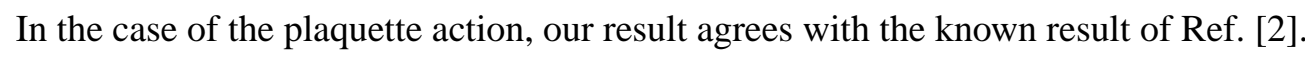

It is worth noting that the value of $Z_{1}$ (and of $e_{3}$, see below) for the DBW2 action is the smallest one, leading to a renormalization factor $Z_{Q}$ closer to 1 (and $M\left(g^{2}\right)$, Eq. (3.2), closer to 0). This would single out the DBW2 action as a better candidate for studies of topology.

\section{Computation of $\mathbf{M}\left(g^{2}\right)$}

The second task we attend to is the calculation of the additive renormalization of the topological charge susceptibility, which is defined as

$$
\chi_{L}=\sum_{x}\left\langle q_{L}(x) q_{L}(0)\right\rangle
$$

$\chi_{L}$ develops an unphysical background term which becomes dominant in the continuum limit

$$
\chi_{L}\left(g^{2}\right)=a^{4} Z_{Q}\left(g^{2}\right)^{2} \chi+M\left(g^{2}\right), \quad M\left(g^{2}\right)=e_{3} \cdot g^{6}+e_{4} \cdot g^{8}+\cdots
$$




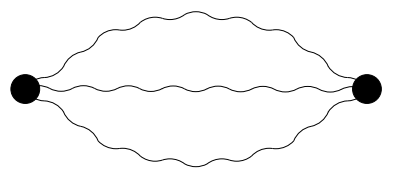

Figure 2: 2-loop diagram contributing to $e_{3}$. Bullets stand for topological charge vertices.

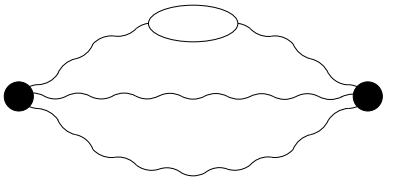

(a)

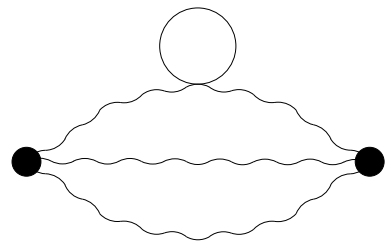

(b)

Figure 3: Diagrams contributing to $e_{4}^{f}$. Wavy (straight) lines correspond to gluons (overlap/clover fermions).

$M\left(g^{2}\right)$ is the power divergent additive renormalization of $\chi_{L}$. We compute the 2-loop coefficient $e_{3}$. This quantity is evaluated for several sets of values of the Symanzik improvement coefficients. Figure 2 shows the diagram contributing to $e_{3}$.

The 3-loop term $e_{4}$ of the expansion of $M\left(g^{2}\right)$ equals $e_{4}=e_{4}^{g}+e_{4}^{f}$, where $e_{4}^{f}$ stands for the fermionic contribution to $e_{4}$ ( $c_{\mathrm{SW}}$ is the coefficient in the clover action)

$$
e_{4}^{f}=N_{f}\left(N_{c}^{2}-1\right) N_{c} \cdot\left(e_{4,0}+e_{4,1} c_{\mathrm{SW}}+e_{4,2} c_{\mathrm{SW}}^{2}\right)
$$

and $e_{4}^{g}$ is the purely gluonic contribution, expressed as in Ref. [0]

$$
e_{4}^{g}=\frac{1}{16}\left(N_{c}^{2}-1\right)\left(1.735 N_{c}^{2}-10.82+73.83 / N_{c}^{2}\right) \times 10^{-7}
$$

In fact, what we are interested in, is the calculation of the parameters $e_{4,0}, e_{4,1}, e_{4,2}$. This task is performed using both overlap and clover fermions (clearly, overlap fermions involve only the parameter $e_{4,0}$ ). Figure 3 shows the 3-loop diagrams contributing to the evaluation of $e_{4}^{f}$.

The propagator and vertices of overlap fermions can be obtained from the following expression for the overlap action, written in terms of the massless Neuberger-Dirac operator $D_{N}$ [10]

$$
S_{\text {Overlap }}=a^{4} \sum_{n, m} \bar{\Psi}(n) D_{N}(n, m) \Psi(m), \quad D_{N}=\frac{M_{0}}{a}\left(1+\frac{X}{\sqrt{X^{\dagger} X}}\right)
$$

$M_{0}$ is a real parameter corresponding to a negative mass term. $M_{0}$ must lie in the range $0<M_{0}<2 r$, $\mathrm{r}$ being the Wilson perameter (in our case $r=1$ ). $X$ is the Wilson-Dirac operator with mass $-M_{0}$.

The clover (SW) fermionic action [11], contains an extra term, parameterized by a coefficient, $c_{\mathrm{SW}}$; this coefficient is treated here as a free parameter.

In performing this calculation, a large effort was devoted to the creation of an efficient 3-loop "integrator", that is, a metacode for converting lengthy 3-loop integrands into efficient code for numerical integration. The output code of the integrator precalculates a number of time-consuming common ingredients (Symanzik propagator, overlap expressions, etc.), exploits symmetries of the 
integration region, integrates in parallel over non-overlapping loops, organizes the integrand as an inverse tree for optimized evaluation of innermost loops, etc.

Table 1 contains our results for $e_{3}$ (cf. Eq.(3.2) for different gluonic actions. These results, for the case of the plaquette action, agree with older known results (see, e.g., [12]). Figure 4 shows the coefficients $e_{4,0}, e_{4,1}, e_{4,2}$ of the clover result for different values of the bare fermion mass $m$. Figure 5 exhibits the dependence of $e_{4}$, using the overlap action, on the parameter $M_{0}$.

A complete tabular version of our results on $e_{4}$, for both the clover and overlap cases, can be found in our longer write-up, Ref. [1]].

\begin{tabular}{llllll}
\hline \hline \multicolumn{1}{c}{$c_{0}$} & \multicolumn{1}{c}{$c_{1}$} & \multicolumn{1}{c}{$c_{3}$} & \multicolumn{1}{c}{$Z_{11}$} & \multicolumn{1}{c}{$Z_{12}$} & \multicolumn{1}{c}{$e_{3} \times 10^{-7}$} \\
\hline \hline 1.0 & 0.0 & 0.0 & $-0.33059398205(2)$ & $0.2500000000(1)$ & $6.89791329(1)$ \\
1.6666666 & -0.083333 & 0.0 & $-0.2512236240(1)$ & $0.183131339233(1)$ & $3.1814562840(7)$ \\
2.3168064 & -0.151791 & -0.0128098 & $-0.20828371039(3)$ & $0.147519438874(3)$ & $1.8452250005(2)$ \\
2.3460240 & -0.154846 & -0.0134070 & $-0.20674100461(1)$ & $0.146259768983(1)$ & $1.8054229585(4)$ \\
2.3869776 & -0.159128 & -0.0142442 & $-0.20462181183(1)$ & $0.144531861677(4)$ & $1.7516351593(8)$ \\
2.4127840 & -0.161827 & -0.0147710 & $-0.20331145580(1)$ & $0.143464931830(1)$ & $1.7188880608(5)$ \\
2.4465400 & -0.165353 & -0.0154645 & $-0.20162651307(1)$ & $0.142094444611(2)$ & $1.6773505020(9)$ \\
2.4891712 & -0.169805 & -0.0163414 & $-0.19954339172(1)$ & $0.140402610424(1)$ & $1.626880218(1)$ \\
3.648 & -0.331 & 0.0 & $-0.15392854668(1)$ & $0.105132852383(2)$ & $0.752432061(7)$ \\
12.2688 & -1.4086 & 0.0 & $-0.0617777059(4)$ & $0.038277296152(6)$ & $0.04881939(4)$ \\
\hline \hline
\end{tabular}

Table 1: The values of $Z_{11}$ and $Z_{12}$ (Eq.(2.2), Figure 1), and of $e_{3}$ (Eq.(3.2), Figure 2), with Symanzik improved gluons, for various values of the coeffi cients $a_{0}, c_{1}, c_{3} \cdot\left(c_{2}=0\right)$

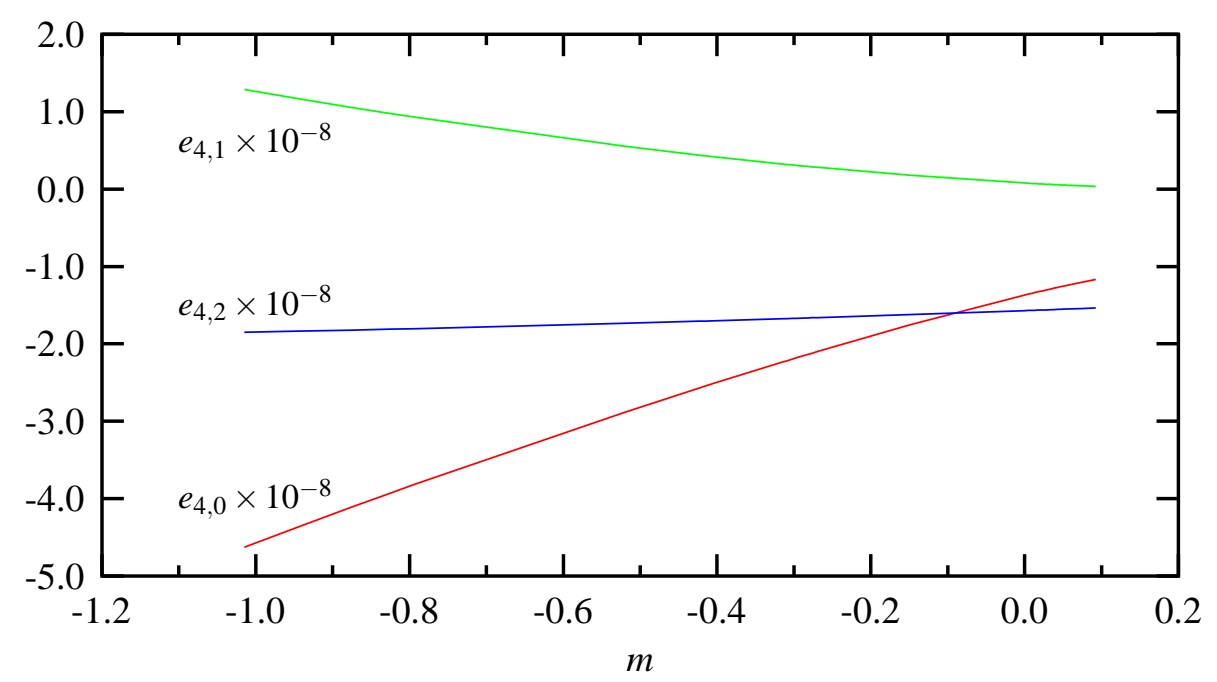

Figure 4: Variation of the terms contributing to $e_{4}^{f}$ as a function of $m$ 


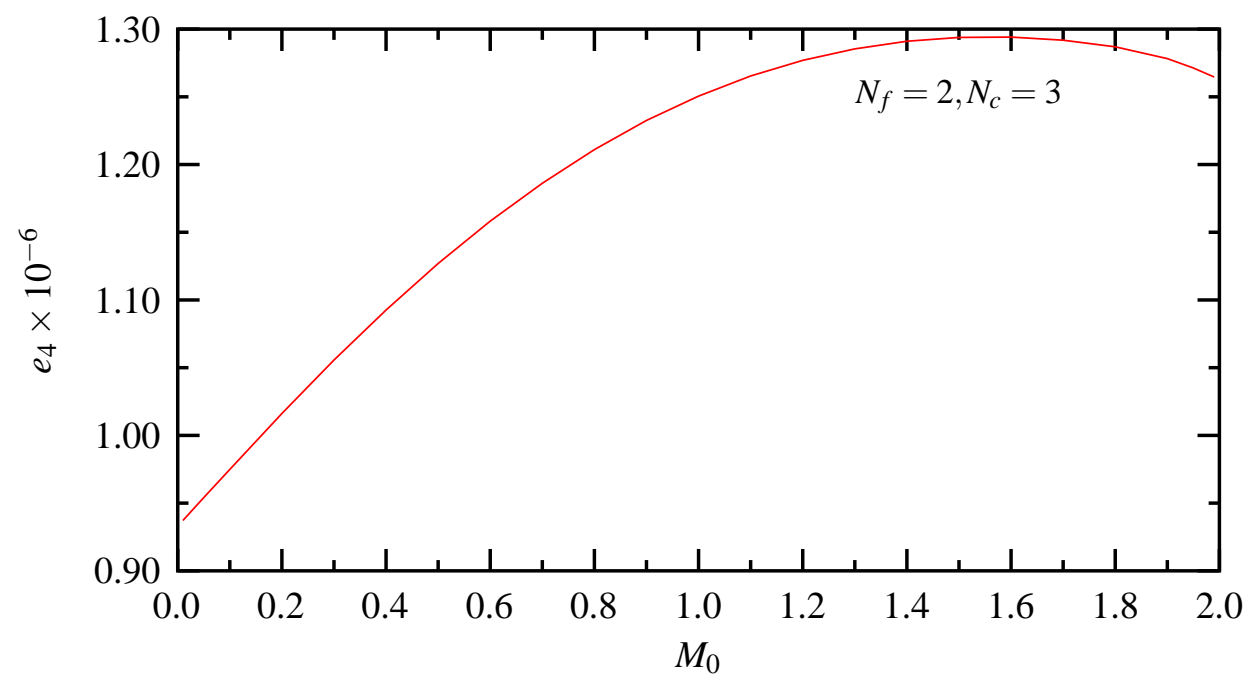

Figure 5: Value of $e_{4}$ as a function $M_{0}$

\section{References}

[1] A. Skouroupathis and H. Panagopoulos, Additive and multiplicative renormalization of topological charge with improved gluon/fermion actions: A test case for 3-loop vacuum calculations, using overlap or clover fermions, hep-lat/0509012.

[2] M. Campostrini, A. Di Giacomo and H. Panagopoulos, The topological susceptibility on the lattice, Phys. Lett. B 212 (1988) 206.

[3] H. Panagopoulos and E. Vicari, The trilinear gluon condensate on the lattice, Nucl. Phys. B332 (1990) 261.

[4] R. Horsley et al., One-loop renormalisation of quark bilinears for overlap fermions with improved gauge actions, Nucl.Phys. B693 (2004) 3 [Erratum-ibid. B713 (2005) 601].

[5] K. Symanzik, Continuum limit and improved action in lattice theories. 1. Principles and $\phi^{4}$ theory, Nucl. Phys. B226 (1983) 187.

[6] M. G. Alford, W. Dimm, G. P. Lepage, G. Hockney and P. B. Mackenzie, Lattice QCD on small computers, Phys. Lett. B 361 (1995) 87.

[7] Y. Iwasaki, Renormalization group analysis of lattice theories and improved lattice action. 2. Four-dimensional nonabelian SU(N) gauge model, UTHEP-118 (1983).

[8] T. Takaishi, Heavy quark potential and effective actions on blocked configurations, Phys. Rev. D54 (1996) 1050.

[9] B. Allés, M. Campostrini, A. Feo and H. Panagopoulos, Lattice perturbation theory by computer algebra: A three loop result for the topological susceptibility, Nucl. Phys. B413 (1994) 553.

[10] H. Neuberger, Exactly massless quarks on the lattice, Phys. Lett. B 417 (1998) 141; More about exactly massless quarks on the lattice, Phys. Lett. B 427 (1998) 353.

[11] B. Sheikholeslami and R. Wohlert, Improved continuum limit action for QCD with Wilson fermions, Nucl. Phys. B259 (1985) 572.

[12] C. Christou, A. Di Giacomo, H. Panagopoulos and E. Vicari, Improved lattice operators: The case of the topological charge density, Phys. Rev. D53 (1996) 2619. 